\title{
Diabetischer Fuß als Problemfeld
}

\author{
Mangelndes Krankheitsgefühl der Patienten macht Behandlung schwierig
}

Der Patient mit diabetischem Fußsyndrom ist ein schwieriger Patient. Die Schwere der Erkrankung ist ihm nicht bewusst. Er spürt weder Druck noch Wunde und stellt damit keine Verbindung zwischen dem geschädigten Bein und sich selbst her. Geduld und Hartnäckigkeit sind daher in der Behandlung gefordert.

Etwa 20 Prozent der Diabetiker erleiden im Verlauf ihrer Erkrankung ein Diabetisches Fußsyndrom (DFS) in Form von Haut- und Nagelproblemen und Deformationen bis hin zur Wunde, lokalisiert oft auf der Fußsohle. Die Rezidivrate ist ziemlich hoch und beträgt nach einem Jahr 34, nach fünf Jahren 70 Prozent. Mit der hohen Rezidivrate steigt auch das Risiko für eine Amputation und in der Folge die Mortalität. Dennoch: Trotz dieser schlechten Aussichten, ist der Patient nur schlecht zur Mitarbeit zu motivieren. Die häufig durch die Diabeteserkrankung verursachte Schädigung der Nerven in Form einer Polyneuropathie verhindert, dass er all diese Schäden spürt. „Sie nehmen die Schwere ihres Defekts nicht wahr", stellte Anita Mysor, Wundmanagerin in Krefeld und Berlin, Ende Jänner in Wien in einem Workshop von Lohmann\&Rauscher zum Thema „Innovative Wundtherapie - Herausforderung Diabetisches Fußsyndrom", fest: „Dessen muss sich der Therapeut bewusst sein und das macht die Therapie so schwer."

\section{Unbekehrbar und immer fröhlich}

Wichtig in der Betreuung des Diabetespatienten sind die frühe Diagnostik und Prävention und die Schulung. Die ersten Hinweise auf Probleme sind sichtbar: Die Füße sind sehr trocken, es kommt zu Schwielenbildung, Nagelpilz und Ausbildung eines Hallux. Die typischen Stellen befinden sich auf der Fußsohle, daher kommt auch der Schuhauswahl große Bedeutung zu. Die fehlende Empfindsamkeit führt bei den Patienten häufig dazu, dass Schuhe zu klein gekauft werden, die Druckstellen erhöhen freilich die Gefahr von Läsionen. „Patienten mit Diabetischem Fußsyndrom sind anders", fasste Mysor zusammen: „Befund und Erleben der Patienten sind weit auseinander - die Patienten sind subjektiv beschwerdefrei, sie sind daher uneinsichtig und verständnislos gegenüber dem therapeutischen Vorgehen - und sie sind immer fröhlich!“

\section{Lokale Wundversorgung in vielen Varianten}

Wie bei allen chronischen Wunden ist auch beim diabetischen Fußulkus die feuchte Wundbehandlung der trockenen Wundversorgung vorzuziehen. Allerdings, so Mysor, gebe es keine Leitlinie, wann was anzuwenden sei und die Entscheidung daher sehr stark von der persönlichen Erfahrung und Intuition geprägt. Darüber hinaus müsse der Verband zusätzlich zum Infektions- und Druckschutz auch dazu dienen, den Patienten vor sich selbst zu schützen - da der Patient seine eigene Gefährdung meist nicht erkennt. Einer der Vorteile der modernen Wundauflagen sei auch die längere Verweildauer auf der Wunde, wodurch weniger Verbandwechsel notwendig sind.

Der relativ harmlose Beginn des DFS und die späte Diagnostik führen zu einer hohen Rate an Spätkomplikationen wie Ulzerationen und auch Amputationen, betonte Dr. Claudia Ellert, Gefäßchirurgin an den Lahn-Dill-Kliniken in Wetzlar. Die Therapie soll diabetische Fußläsionen möglichst verhindern und die Durchblutung verbessern. Dafür sei eine engmaschige Anbindung der spezialisierten Zentren an Hausärzte und Diabetologen notwendig, so Ellert.

$\mathrm{Zu}$ den Zielen der Therapie des DFS zählen die Infektionskontrolle, die arterielle Revaskularisation, die Ulkusabheilung, die Schmerzreduktion, Wiederherstellung der Gehfähigkeit, Mobilität und Selbstständigkeit. Die Wundsituation, mit der das Therapeutenteam im Krankenhaus konfrontiert sei, sei durch die Infektion oft bereits schwierig. Hier hat sich bei komplexen Wundsituationen die Unterdrucktherapie in Form von computerassistierten vakuumgestützten Systemen als Standard durchgesetzt. Durch das genau steuerbare Vakuum kommt es zur Zelldehnung und zu einem Reiz für die Granulation und Proliferation. Als Nebeneffekt wird die lokale Durchblutung ge-

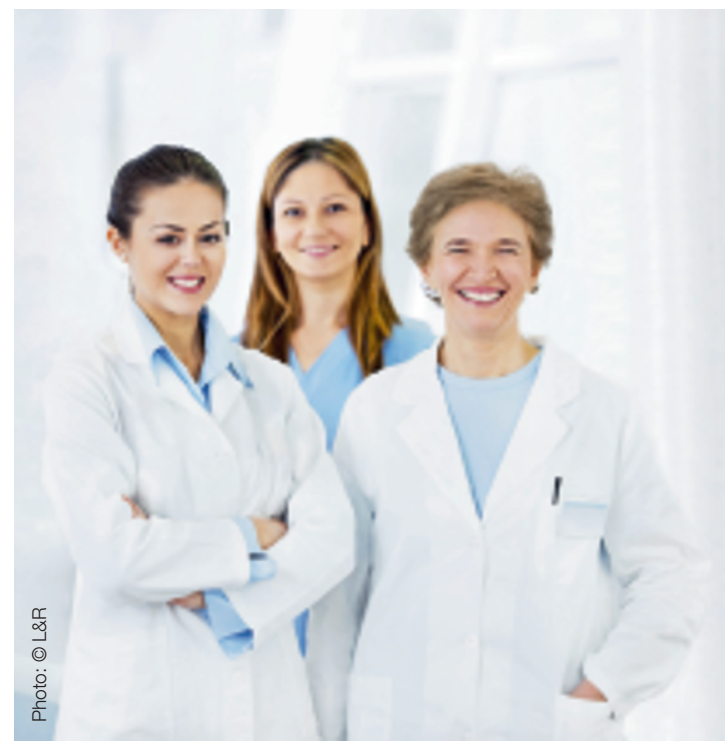

Im Behandlungsteam zum Behandlungserfolg.

steigert, es kommt zu einer Ödemreduktion, die Folienabdeckung führt zur Keimreduktion und die vakuumbedingte Annäherung der Wundränder führt auch zur Verkleinerung der Wundfläche.

Untersuchungen zur Unterdrucktherapie zeigen gegenüber der herkömmlichen Therapie eine positive Wirkung, berichtete Ellert: „Die Dauer bis zur Heilung wird verkürzt und damit auch jene der Krankenhausaufenthalte, die Vakuumtherapie macht komplexe Wunden versorgbar, sie ist einfacher handzuhaben, vermeidet eine beträchtliche Zahl an Amputationen und erhöht die Lebensqualität." Moderne Wundversorgung in einem interdisziplinären Netzwerk kann die Therapie des DFS also wesentlich verbessern - mit entsprechender Geduld und Verständnis für die Betroffenen. $k i$

Quelle: „L\&R Update: Innovative Wundtherapie Herausforderung Diabetisches Fußsyndrom“, 27. Jänner 2016, Wien.

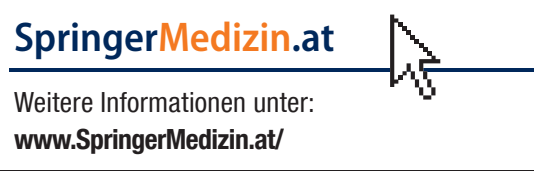

